\title{
Comportamento de restrição alimentar e obesidade
}

\author{
Restrained eating behavior and obesity
}

\author{
Fabiana BERNARDI' \\ Cristiane CICHELERO ${ }^{1}$ \\ Márcia Regina VITOLO²
}

\section{R E S U M O}

A obesidade é uma doença de alta prevalência no mundo e é responsável por sérias repercussões orgânicas e psicossociais, desde a infância até a vida adulta. O comportamento alimentar tem bases biológicas e sociais e, associado, à obesidade, torna-se um processo ainda mais complexo pelos aspectos psicológicos envolvidos, os quais se expressam por meio de humor depressivo, ansiedade, sentimento de culpa e, também, por mecanismos fisiológicos, como a resistência ao jejum na vigência de dietas restritivas. Há evidências de que, em indivíduos obesos, comportamentos de compulsão alimentar e ou restrição são mais freqüentes e parecem ser, em parte, responsáveis pelos fracassos observados no tratamento da obesidade. As restrições e auto-imposições das pessoas que fazem dieta, parecem ter um efeito rebote, resultando em compulsão alimentar, a qual pode associar-se a conseqüências psicológicas, como a perda da auto-estima, mudanças de humor e distração. As reflexões desta revisão sugerem que os programas para redução de peso corporal devem enfocar as bases do comportamento alimentar e desenvolver, efetivamente, ações interdisciplinares que permitam obter resultados eficazes no tratamento da obesidade.

Termos de indexação: conduta alimentar, obesidade, peso corporal, restrição alimentar.

\section{A B S T R A C T}

Obesity is a widespread disease in the world, responsible for serious organic and psychosocial repercussions, from infancy to adulthood. Eating behavior has biological as well as social bases. Associated to obesity, it becomes an even more complex process, since it is accompanied by psychological aspects showing symptoms

\footnotetext{
1 Nutricionistas, Curso de Especialização em Nutrição Clínica, Centro de Ciências da Saúde, Universidade do Vale do Rio dos Sinos. São Leopoldo, RS, Brasil.

2 Programa Interdisciplinar de Promoção e Atenção à Saúde, Universidade do Vale do Rio dos Sinos. Caixa Postal 551 Agência Unisinos, 93022-970, São Leopoldo, RS, Brasil. Correspondência para/Correspondence to: M.R. VÍTOLO. E-mail: <vitolo@bios.unisinos.br>.
} 
such as depressive moods, anxiety, feelings of guilt, and physiological mechanisms as, for instance, the resistance to fast during restrictive diets. Obese individuals prevalently present behaviors such as binge or restrained eating, both leading to failure in the treatment of obesity. Restrained eating refers to the tendency to restrict food intake consciously, in order to prevent weight gain or to promote weight loss, which may be associated to episodes of overeating and have psychological consequences as, for example, the loss of self-steam, mood distress and distraction. The results of this review suggest that weight control programs should focus on the bases of eating behaviors, and, effectively, develop interdisciplinary courses of action in order to obtain positive results in the treatment of obesity.

Indexing terms: adaptation, psychological, feeding behavior, obesity, body weight, restrained eating.

\section{N T R O D U Ç Ã O}

A obesidade emergiu como uma epidemia em países desenvolvidos, durante as últimas décadas do século XX. No entanto, atualmente, atinge todos os níveis socioeconômicos e vem aumentando sua incidência, também nos países em desenvolvimento. A obesidade não está limitada a uma região, país ou grupo racial/étnico. A obesidade é um fenômeno mundial que afeta ricos e pobres e é resultante da ação de fatores ambientais (hábitos alimentares, atividade física e condições psicológicas) sobre indivíduos geneticamente predispostos a apresentar excesso de tecido adiposo'.

Devido à sua etiologia multifatorial é difícil mensurar a força de cada uma das variáveis envolvidas no processo do ganho excessivo de peso. Entre elas, destaca-se a influência dos fatores socioculturais que impõem um padrão de beleza esbelto em que as mulheres, especialmente, vivem de acordo com a tirania da moda, contrariando suas necessidades nutricionais. Controlar a ingestão alimentar por meio de dietas restritivas e tornar crônico esse comportamento devido à pressão sociocultural, que impõe padrões corporais cada vez mais magros, são atitudes possivelmente desencadeadoras de transtornos alimentares ${ }^{2,3}$. É interessante destacar que também há o contraponto desse padrão de beleza em alguns países denominados pré-industrializados, com pouca disponibilidade de alimentos, em que ser gordo é um símbolo de status até hoje4 .

A obesidade é uma doença de difícil controle, com altos percentuais de insucessos terapêuticos e de recidivas, podendo apresentar sérias repercussões orgânicas e psico-sociais, especialmente nas formas mais graves.

A teoria cognitiva social, proposta por Bandura, citado por Baldwin \& Falciglia ${ }^{5}$, descreve o comportamento humano como sendo determinado por disposições internas e influências ambientais. Comportamento, fatores pessoais internos e eventos ambientais interagem entre si. Métodos cognitivos comportamentais modificam sentimentos e ações, influenciando o padrão de pensamento das pessoas. Segundo Rollnick ${ }^{6}$, mudanças no comportamento alimentar e na atividade física constituem processos ativos nos quais as pessoas têm de se esforçar, consciente e consideravelmente, a fim de mudar antigos hábitos. O comportamento alimentar envolve $\mathrm{O}$ apetite (sensação de fome e saciedade), os estados motivacionais e a necessidade de ingestão energética (processos fisiológicos e metabólicos), coordenados pela atividade dos sistemas nervosos periférico e central (vias neurais e receptores) ${ }^{7}$.

A abordagem desta revisão pretendeu enfatizar as bases e os processos que norteiam o comportamento de restrição alimentar e sua interface com a obesidade. Para subsidiar a discussão, foram resgatados alguns aspectos do comportamento alimentar, assim como aspectos psicológicos envolvidos com a questão, que dificultam o tratamento para redução de peso.

\section{Aspectos psicológicos na obesidade}

O indivíduo obeso apresenta sofrimento psicológico decorrente tanto dos problemas 
relacionados ao preconceito social e à discriminação contra a obesidade, como das características do seu comportamento alimentar. A depreciação da própria imagem física leva à preocupação opressiva com a obesidade, tornando o obeso inseguro devido à sua inabilidade de manter a perda de peso. A falta de confiança, a sensação de isolamento, atribuída ao fracasso da família e dos amigos em entender o problema, assim como a humilhação, decorrente do intenso preconceito e discriminação aos quais os indivíduos obesos estão sujeitos, remetem enorme carga psicológica ao obeso ${ }^{8}$. Entretanto, importa enfatizar que, entre as pessoas com excesso de peso, não foi demonstrada nenhuma condição psicológica própria, nem traços de personalidade comuns ${ }^{9}$.

Há evidências de que grande parte dos indivíduos obesos, come para resolver ou compensar problemas dos quais, às vezes, não têm consciência. Esses mesmos indivíduos podem apresentar dificuldades em obter prazer nas relações sociais, por se sentirem rejeitados ou discriminados, o que os leva ao isolamento. Por outro lado, esses sentimentos contribuem para que os obesos enxerguem a comida como importante fonte de prazer, o que, devido ao preconceito, conseqüentemente, restringe e empobrece ainda mais suas relações afetivas e sociais. Esse processo alimenta o ciclo vicioso em que, de um lado, há o ganho progressivo de peso e, do outro, uma solidão cada vez maior ${ }^{10}$.

A ingestão alimentar, em diferentes condições emocionais, parece ocorrer mais evidentemente na vigência de excesso de peso, pois os indivíduos obesos consomem mais alimentos em situação de estresse emocional. Essa teoria, chamada de Modelo Psicossomático da Obesidade ${ }^{11}$, afirma que as pessoas obesas, principalmente do gênero feminino, comem excessivamente como mecanismo compensatório em situações de ansiedade, depressão, tristeza, raiva $^{12,13}$. Associando esse comportamento às características sociais de nossa época, tais como a lipofobia, a obsessão por ser magro e a conseqüente rejeição da obesidade, grande parte da população obesa sonha ser esbelta, vivendo e sofrendo essa contradição ${ }^{14}$.

Entre as pessoas que buscam tratamento para controle de peso, a incidência de compulsão alimentar aumentou significativamente ${ }^{15}$. A prevalência de compulsão alimentar varia de $20 \%$ a $50 \%$, entre indivíduos obesos participantes de programa de redução de peso ${ }^{16,17}$.

De acordo com o Manual Diagnóstico e Estatístico de Transtornos Mentais da Associação Americana de Psiquiatria, $4^{\text {a }}$ edição (DSM-IV) ${ }^{18}$, a compulsão alimentar é um transtorno alimentar que inclui indivíduos que comem descontroladamente, em um intervalo de tempo máximo de duas horas, devido ao estresse ou a sentimentos negativos, uma quantidade de comida que, definitivamente, é maior do que a quantidade que a maioria das pessoas comeria em um período de tempo semelhante. Durante o episódio, fica evidente o sentimento de perda do controle do próprio comportamento.

As pessoas que apresentam o transtorno do comer compulsivo têm ataques bulímicos repetidos, mas não evidenciam as medidas patológicas de controle de peso que os pacientes com bulimia nervosa utilizam, como os comportamentos compensatórios (vômitos, abuso de laxativos, exercício físico excessivo) que sucedem o episódio bulímico. Durante o episódio, o indivíduo sente como se não tivesse nenhuma possibilidade de controlar sua atitude compulsiva alimentar e, após o episódio, refere desconforto psicológico ${ }^{19}$. Há evidências epidemiológicas de que o início de dietas ocorre, geralmente, após o aparecimento dos ataques de comer compulsivo, caracterizando a distinção entre este comer compulsivo e o quadro de bulimia, no qual os ataques se sucedem às dietas ${ }^{16,20}$.

Comedores compulsivos obesos, freqüentemente, preocupam-se com a forma e o peso corporal e em fazer dieta; possuem imagem corporal negativa e fortes impulsos para comer em excesso ${ }^{21}$. Sofrem maior angústia psicológica do que obesos não compulsivos, como baixa auto-estima, altos níveis de depressão e outras 
desordens psiquiátricas, como ansiedade, síndrome do pânico e transtornos da personalidade ${ }^{17,22}$. Os indivíduos com este padrão alimentar, geralmente, apresentam variados graus de obesidade e um histórico de flutuações ponderais mais acentuadas do que obesos sem compulsão alimentar. Alguns continuam nas tentativas de restrição energética e, devido aos fracassos repetidos, abandonam quaisquer tentativas de fazer dietas ${ }^{23}$. A obesidade, por si só, remete a riscos para a saúde e, quando acompanhada de compulsão alimentar, estes riscos são ampliados. $\mathrm{Na}$ vigência desse quadro, o tratamento mais adequado seria centrar-se, inicialmente, na diminuição da freqüência de compulsão alimentar e, posteriormente, no emagrecimento ${ }^{24}$.

A influência dos aspectos socioculturais é marcante. Considerando a pressão social da busca pela magreza e todos os esforços envolvidos em submeter-se a um tratamento, trava-se uma guerra entre corpo e mente. Estudos mostram que estresse, restrição, rejeição e outros estados emocionais negativos, levam à interrupção de autocontrole, conduzindo, assim, a escolhas alimentares inapropriadas, as quais, de certa forma, aliviam as tensões vigentes ${ }^{25}$.

\section{Comportamento alimentar}

Muitos pacientes obesos referem ingestão alimentar compatível com os parâmetros da normalidade. Alguns, involuntariamente, ocultam parcela significativa do volume energético efetivamente ingerido. É extremamente difícil medir a quantidade de energia consumida. Pesquisa mostrou, em revisão, que $70 \%$ dos obesos informam valores fisiologicamente improváveis de energia ingerida ${ }^{26}$.

French et al. ${ }^{27}$, por meio da observação de vários estudos, concluíram que as pessoas com comportamento de fazer dieta (dieters), não necessariamente obesas, possuem relatos de baixa ingestão de alimentos energéticos e ricos em gordura, como doces, carnes, batata frita e refrigerante, e alta freqüência de atividade física intensa, comparadas àquelas que não têm o comportamento de controlarem o que comem. Esse quadro é reflexo de um viés metodológico para estimar o consumo alimentar, especialmente a ingestão energética, já que alguns indivíduos, repetidamente, relatam valores incompatíveis com a manutenção do peso, não havendo evidências da existência de indivíduos que, efetivamente, consomem pouco e mantêm, no longo prazo, o mesmo padrão corporal28-31. As mulheres apresentam com mais freqüência comportamento de sub-relato alimentar, devido às maiores pressões culturais e sociais a que são submetidas para manter hábitos alimentares saudáveis e padrão corporal esguio ${ }^{32,33}$.

O controle de energia consumida está baseado em um conjunto de interações que formam a psicobiologia do comportamento alimentar, envolvendo o apetite, os processos fisiológicos e metabólicos, e o sistema nervoso. A busca por comida, surgida da necessidade do processo metabólico, é determinada por sistemas sensórios específicos, associados ao cheiro e gosto. Blundell \& Gillett ${ }^{26}$ descrevem as seguintes influências sobre o controle do comportamento alimentar, o que explica, em parte, a dificuldade de obter dados mais coerentes entre consumo alimentar e níveis de gasto energético de indivíduos adultos, especialmente daqueles com excesso de peso:

a) processos biológicos que refletem os desejos de comer e processos inibitórios de ingestão alimentar e reserva de tecido adiposo;

b) processos ambientais que restringem 0 tempo e a distribuição de episódios alimentares, influenciando o consumo energético por meio do sabor e do conteúdo de nutrientes dos alimentos;

c) modulações auto-impostas do padrão de comportamento, que surge da interação entre processos biológicos e ambientais;

d) a tentativa comportamental de autocontrole é, freqüentemente, incerta, porque tende a se opor a tendências biológicas e pressões ambientais. 
Dois terços dos obesos consomem carboidratos, não somente para aliviar a fome, mas para combater tensões, ansiedade, fadiga mental e depressão. Estudos ${ }^{34,35}$ demonstraram que dietas ricas em carboidratos influenciam o apetite, ou seja, na fase pós-prandial, os carboidratos promovem sua própria oxidação, estimulando a secreção de insulina e captação de glicose celular. Se a dieta contém pouco carboidrato, pode ocorrer aumento da fome. Depois de uma dieta rica em carboidrato, a oxidação de glicose aumenta e a sensação de saciedade se eleva. Na fase pós-prandial tardia, quando os estoques de glicogênio diminuem, a oxidação de glicose diminui e a sensação de fome começa a aumentar, os mecanismos que retardam esse processo de esgotamento de glicogênio aumentam o período de saciedade.

Há evidências de que a gordura dietética pode enfraquecer a regulação de energia consumida, isto é, refeições ricas em lipídios reduzem níveis de leptina no plasma por 24 horas, o que pode desencadear a liberação do neuropeptídeo, o qual tem o papel de aumentar a ingestão alimentar. Conseqüentemente, o consumo de alimentos ricos em lipídios contribui diretamente para o equilíbrio energético positivo, por aumentar a fome e quantidade de energia consumida, o que conduzirá ao ganho de peso. Há maior incidência de obesidade entre consumidores habituais de alimentos ricos em lipídios, do que entre consumidores de alimentos com baixo teor de lipídios. Mulheres obesas mostram uma forte preferência por alimentos doces, ricos em gordura ${ }^{26}$.

Hess $^{36}$ afirmou que, no processo evolutivo, o cérebro foi programado a procurar substâncias de sabor doce, como fonte de nutrientes e energia. Receptores do sabor doce transmitem uma mensagem ao cérebro, que libera endorfinas, o "ópio" corporal. O aumento da liberação deste neurotransmissor provoca saciedade. Talvez, isto explique porque, em períodos de tensão e ansiedade, muitas pessoas procuram substâncias doces.

\section{Restrição alimentar}

Restrição alimentar é uma estratégia comportamental e cognitiva, que as pessoas usam para controlar o peso corporal ${ }^{37}$.

O termo restrained eating foi definido como uma tendência a restringir o consumo alimentar conscientemente, a fim de prevenir o ganho de peso ou promover sua perda. Estudo envolvendo 387 famílias francesas, num total de 1320 indivíduos entre 11 e 65 anos, objetivou descrever os padrões alimentares de famílias francesas e avaliar a relação entre consumo dietético, perfil alimentar e sobrepeso. Os resultados mostraram que a restrição alimentar foi significativamente relacionada com excesso de peso e menor ingestão energética em homens e mulheres. Em mulheres, foi associada à menor ingestão de proteínas e carboidratos $^{38}$.

Estudos demonstraram que unrestrained eaters, pessoas que não restringem o consumo de alimentos, ou nondieting, pessoas que não fazem dieta, comem menos após um lanche altamente energético. Esta regulação de energia é normal, isto é, a pessoa compensa o lanche comendo menos, posteriormente. Porém, restrained eaters, pessoas que restringem 0 consumo alimentar, comem mais depois de um lanche altamente energético. Este comportamento é explicado pela desinibição do controle cognitivo, uma vez que acreditam ter excedido o consumo alimentar permitido com a ingestão do lanche energético. Esta situação pode resultar em episódios de compulsão alimentar, alternados a períodos de restrição energética ${ }^{39,40}$.

Estudo realizado por Westenhoefer ${ }^{41}$, com 50 mil homens e mulheres, participantes de um programa para perda de peso, mostrou que a alta pontuação inicial de restrição alimentar está associada à maior ingestão alimentar e maior Índice de Massa Corporal (IMC). Estes resultados talvez se devam às fracassadas tentativas de perder ou manter o peso e, provavelmente, indicam dificuldades no controle alimentar e preocupações com a forma corporal. 
Foi demonstrado que subestimar a ingestão alimentar é particularmente predominante entre indivíduos obesos ou dieters (aqueles que estão sempre fazendo dieta), os quais tentam impor algum controle sobre o próprio padrão alimentar e seleção de alimentos, coagidos pela pressão social adversa ao ganho de peso e à obesidade. Esta atitude é, comumente, denominada restrição alimentar e, geralmente, vem seguida pela disinhibition. Entende-se a "desinibição", neste caso, como tendência de indivíduos reprimidos a liberarem a ingestão energética, em resposta a determinados estímulos. O consumo alimentar durante a disinhibition pode ser muito grande, tendendo a constituir episódios de compulsão alimentar, pois é muito difícil sustentar a restrição energética por muito tempo ${ }^{26}$.

Polivy ${ }^{40}$ explicou que o regime de inanição e a auto-imposição das pessoas que fazem dieta, parecem resultar em compulsão alimentar e em manifestações psicológicas, como preocupação com comida e em comer, aumento da responsabilidade emocional e mudanças de humor e distração. As conseqüências não são tanto a privação alimentar no longo prazo, mas a privação psicológica causada por evitar determinados alimentos ou por, eventualmente, tentar restringir o consumo total energético.

Diferenças fisiológicas também foram encontradas no comportamento de restrição alimentar. A percepção do sabor diferiu entre as pessoas famintas, pacientes com anorexia nervosa e as que fazem dieta. As qualidades sensoriais (sabor, cheiro, textura e aparência) são fortes determinantes do comportamento alimentar. Segundo Mattes \& Kare apud Assis \& Nahas ${ }^{42}$, as propriedades sensoriais dos alimentos desempenham papel não somente na determinação do seu consumo, mas também na determinação da saciedade, ingestão e seleção do alimento em uma refeição. Essas considerações confirmam a complexidade do comportamento alimentar na obesidade, sugerindo que o tratamento dietético deve considerar, tanto as motivações psicológicas do indivíduo para o ato de se alimentar, como as implicações fisiológicas decorrentes da proposta dietética.

Os mecanismos fisiológicos que influenciam o comportamento alimentar e impedem a perda de peso em dietas restritivas, podem ser refletidos: 1) em vias oxidativas para utilização da energia necessária; 2) em mudanças repentinas na disponibilidade de glicose sangüínea; 3) e, eventualmente, em neurotransmissores cerebrais, como o neuropeptídeo-Y, que parece ligar-se ao processo metabólico ${ }^{26}$. Hansen ${ }^{43}$ afirmou que a restrição energética produz alterações nas vias intermediárias de disposição de nutrientes, incluindo redução de glicose sangüínea e variações nos níveis de insulina e leptina.

Os seres humanos mantêm o mesmo peso por muitos anos, o que indica que o peso corporal é regulado em torno de um ponto de equilíbrio (set point); porém, alterações extremas do comportamento alimentar podem levar a distúrbios no controle do peso. O sistema de regulação corporal permite equilíbrio de energia positiva, conduzindo a ganho de peso, mas se defende fortemente contra equilíbrios de energia negativa, que ameaçam causar perda de peso ${ }^{26}$. Dietz ${ }^{44}$ afirmou que as escolhas de alimentos, ou adaptações fisiológicas, em respostas a episódios de escassez de comida, poderiam causar aumento da gordura corporal. Esse aspecto biológico deve ser considerado nos tratamentos dietéticos para redução de peso, já que atua como força contrária.

Estudo com 4537 mulheres e 5004 homens, com objetivo de relacionar excesso de peso e "insegurança alimentar" (disponibilidade incerta ou limitada de adquirir alimentos nutricionalmente adequados e seguros), concluiu que o excesso de peso está relacionado às limitações alimentares em mulheres, mas não em homens ${ }^{45}$. Outro estudo mostrou que o excesso de peso estava relacionado com insegurança alimentar em classes de menor renda ${ }^{46}$. Tais resultados sugerem a associação entre o excesso de adiposidade e as condições anteriores de privação alimentar, geralmente, devidas a fatores socioeconômicos. Essa insegurança na obtenção 
contínua e estável de alimentos pode gerar comportamento de risco para o aumento de reserva energética corporal.

Entre as mulheres que vivem sob condições instáveis para obtenção de alimentos (insegurança alimentar), a ingestão é restringida involuntariamente, devido à falta de recursos financeiros para ter acesso à comida ${ }^{47}$. Entre aquelas que possuem maior estabilidade alimentar, ou seja, que têm acesso aos alimentos nutricionalmente adequados, e a disponibilidade para adquiri-los, a ingestão de comida pode ser restringida voluntariamente, para prevenir o ganho de peso ou para manter o peso ${ }^{40}$. Uma possível explicação para a alta incidência de sobrepeso e obesidade, entre mulheres de baixa condição socioeconômica, é o ciclo instável de aquisição de alimentos, ou seja, períodos de abundância alimentar seguidos de períodos de restrição. Essa situação influencia o ganho de peso por causar padrões alimentares desordenados com bases biopsicossociais ${ }^{48}$.

Williams ${ }^{49}$, em revisão da literatura correspondente, afirmou que as pessoas que persistem em dietas de poucas calorias perdem grandes quantidades de peso, em média, 20kg em 12 a 16 semanas. A maioria destes indivíduos, contudo, recuperam o peso dentro de um curto período de tempo. Muitos deles, reiniciam, então, o processo de dieta, recomeçando o ciclo.

Estudo desenvolvido com um grupo de mulheres restrained eaters, realizado em um período de seis meses, revelou que, embora o peso flutuasse diária e semanalmente, estas muIheres não perderam peso durante os seis meses ${ }^{40}$.

Em face à pressão social adversa ao ganho de peso e obesidade, muitos indivíduos, particularmente mulheres, tentam impor algum controle sobre o próprio padrão alimentar e seleção de alimentos, isto é comumente denominada restrição dietética. Freqüentemente, pessoas obesas tentam perder peso, e a natureza de suas respostas emocionais para perda de peso pode ser refletida como uma característica psicológica. Sintomas como humor depressivo, ansiedade, fraqueza e preocupação em comer são predominantes. Estes sintomas podem ser reduzidos por meio de programas baseados na modificação comportamental, na dieta e nos exercícios ${ }^{8}$.

\section{O N CLUSÃ O}

É necessário interromper o ciclo vicioso e avaliar as intensas demandas psicológicas causadas pela história de restrição alimentar e a pressão social para ser magro. Ambos os fatores trabalham sinergicamente: a pressão social pode levar a excessivas dietas, as quais desencadeiam reações psicológicas e fisiológicas que levam à recuperação e ao aumento da obesidade.

A obesidade deve ser considerada uma condição crônica, que requer apoio periódico e reeducação em aspectos comportamentais e exercícios. Ciclos de perda e recuperação de peso têm mostrado aumentar os riscos à saúde e podem estar relacionados ao desenvolvimento das doenças crônicas não transmissíveis.

As reflexões desta revisão sugerem que programas de redução de peso, individuais ou em grupo, tenham abordagem interdisciplinar, de fato, e que enfatizem, junto aos pacientes, a compreensão dos mecanismos biopsicosociais aos quais estão submetidos. Para isso, a estratégia da Teoria Cognitiva Comportamental parece muito útil para bloquear o ciclo vicioso, exaustivamente discutido neste estudo, tornando-se, portanto, uma aliada no tratamento da obesidade.

\section{REFERÊ NCIAS}

1. Popkin BM, Doak CM. The obesity epidemic is a worldwide phenomenon. Nutr Rev. 1998; 56(4): 106-14.

2. Quandt SA. Social and Cultural Influences on Food Consumption and Nutritional Status. In: Shils ME, Olson JA, Shike M, Ross AC. Modern nutrition in health and disease. 9th ed. Baltimore: Williams \& Wilkins; 1999. p.1783-92.

3. Herscovici CR. A escravidão das dietas. Porto Alegre: Artes Médicas; 1997. p.19-24. 
4. Wang MC, Ho TF, Anderson JN, Sabry IZ. Preference for thinness in Singapure-a newly industrialized society. Singapure Med J. 1999; 40(8):502-7.

5. Baldwin T, Falciglia GA. Application of cognitive behavioral theories to dietary change in clients. J Am Diet Assoc. 1995; 95(11):1315-7.

6. Rollnick S. Behavior change in practice: targeting individuals. Int J Obes Relat Metab Disord. 1996; 20(Suppl I):22S-6S.

7. Netto CA. Psicobiologia do comportamento alimentar. In: Nunes MA, Appolinário JC, Abuchaim AL, Coutinho W. Transtornos alimentares e obesidade. Porto Alegre: Artes Médicas; 1998. p.197-202.

8. Stunkard AJ, Wadden TA. Psychological aspects of severe obesity. Am J Clin Nutr. 1992; 55(2 Suppl): 524S-32S.

9. Sobal J, Devine CM. Social Aspects of Obesity: Influences, Consequences, Assessments and Interventions. In: Dalton S. Overweigth and weight management. Maryland: Aspen Publishers; 1997. p.312-31.

10. Campos ALR. Aspectos psicológicos da obesidade. In: Nóbrega FJ. Distúrbios da nutrição. Rio de Janeiro: Revinter; 1998. p.394-5.

11. Faith MS, Allison, DB, Geliebter, A. Emotional eating and Obesity: Theoretical considerations and practical recommendations. In: Dalton S. Overweigth and weight management. Maryland: Aspen Publishers; 1997. p.439-65.

12. Match M. Characteristics of eating in anger, fear, sadness and joy. Appetite. 1999; 33:129-39.

13. Match M, Gerer J, Ellgring H. Emotions in overweight and normal-weight women, immediately after eating foods differing in energy. Phys Behav. 2003; 80(2-3):367-74.

14. Sant'Anna, DB. Políticas do corpo. São Paulo: Estação Liberdade; 1995.

15. Kalman D, Cascarano H, Krieger DR, Incledon T, Woolsey M. Frequency of binge eating disorder in an outpatient weight loss clinic. J Am Diet Assoc. 2002; 102(5):697-9.
16. Spitzer RL, Yanovski S, Wadden T, Wing R, Marcus $M D$, Stunkard AJ, et al. Binge eating disorder: A multisite field trial for the diagnostic criteria. Int J Eat Disord. 1993; 13(2):137-53.

17. Yanovski SZ,Y Nelson JE, Dubbert BK, Spitzer RL. Binge eating disorder is associated with psychiatric comorbidity in the obese. Am J Psych. 1993; 150(10):1472-9.

18. American Psychiatric Association. Diagnostic and statistical manual of mental disorders. 4th ed. Washington, DC: American Psychiatric Association; 1994.

19. Fairburn CG. Overcoming binge eating. New York: Guilford Press; 1995.

20. Appolinário JC. Transtorno de comer compulsivo. In: Nunes MAA, Apolinario JC, Abuchain ALG, Coutinho W. Transtornos alimentares e obesidade. Porto Alegre: Artmed; 2002. p.40-6.

21. Kuehnel RH, Wadden TA. Binge eating disorder, weight cycling, and psychopathology. Int J Eat Disord. 1994; 15(4):321-29.

22. Telch CF, Agras WS. Obesity, binge eating and psychopathology: Are they related? Int J Eat Disord. 1994; 15(1):53-61.

23. Manual diagnóstico e estatístico de transtornos mentais - DSM-IV. 4a. ed. Porto Alegre: Artes Médicas; 1995.

24. Borges MBF, Jorge MR. Evolução histórica do conceito de compulsão alimentar. In: Psiquiatria na prática médica [ acesso 13 mar 2004]. Disponível em: http://www.unifesp.br/dpsiqu/polbr/ppm/ especial04.htm

25. Goodrick GK, Foreyt JP. Why treatments for obesity don't last. J Am Diet Assoc. 1991; 91(10):1243-7.

26. Blundell JE, Gillett A. Control of food intake in the obese. Obes Res. 2001; 9(4):263S-70S.

27. French SA, Jeffery RW, Murray D. Is dieting good for you? Prevalence, duration and associated weight and behaviour changes for specific weight loss strategies over four years in US adults. Int J Obes Relat Metab Disord. 1999; 23(3):320-7.

28. Fricker J, Baelde D, Igoin-Apfelbaum L, Apfebaum M. Underreporting of food intake in obese "small eaters". Appetite.1992; 19(3):273-83. 
29. French SA, Jeffery RW, Forster JL. Dieting status and its relationship to weight, dietary intake, and physical activity changes over two years in a working population. Obes Res. 1994; 2(2):135-44.

30. Price GM, Paul AA, Cole TJ, Wadsworth MEJ. Characteristics of the low-energy reporters in a longitudinal national dietary survey. $\mathrm{Br} J \mathrm{~N}$ Nutr. 1997; 77(6): 833-51.

31. Black AE. Small eaters or under-reporters? In: Guy-Grand B, Ailhaud G. Progress in obesity research: proceedings of the 8th International Congress on Obesity. London: John Liberty; 1999. p.223-42.

32. Clark D, Tomas F, Withers RT, Brinkman M, Chandler C, Phillips J, et al. Differences in energy metabolism between normal weight "large-eating" and "small-eating" women. Br J Nutr. 1992a; 68(1): 31-44.

33. Clark D et al. Energy metabolism in free-living, "large-eating" and "small-eating" women: studies using ${ }^{2} \mathrm{H}_{2}{ }^{18} \mathrm{O}$. Br J Nutr. 1994b; 72:21-31.

34. Blundell JE, Green S, Burley V. Carbohydrates and appetite. Am J Clin Nutr. 1994; 59(3):728S-34S.

35. Lavin JH, Wittert G, Sun WM, Horowitzl M, Morleyl JE, Read NW. Appetite regulation by carbohydrate: role of blood glucose and gastrointestinal hormones. Am J Physiol Endocrinol Metab. 1996; 271(2 Pt 1):209E-14E.

36. Hess AH. Taste: The neglected nutritional factor. J Am Diet Assoc 1997; 97(10):205S-07S.

37. McGuire MT, Jeffery RW, French SA, Hannan PJ. The relationship between restraint and weight, and weight-related behaviors among individuals in a community weight-gain -prevention trial. Int J Obes Relat Metab Disord. 2001; 25(4):574-80.

38. Lluch A, Herbeth B, Mejean L, Siest G. Dietary intakes, eating style and overweight in the Stanislas Family Study. Int J Obes Relat Metab Disord. 2000; 24(11):1493-9.
39. Pirke KM, Laessle RG. Restrained eating. In: Stunkard AJ, Wadden TA. Obesity: theory and therapy. New York: Raven Press; 1993. p.151-62.

40. Polivy J. Psychological consequences of food restriction. J Am Diet Assoc. 1996; 96(6):589-92.

41. Westenhoefer J. Dietary restraint and disinhibition: is restraint a homogeneous construct? Appetite. 1991; 16(1):45-55.

42. Assis MA, Nahas MV. Aspectos motivacionais em programas de mudança de comportamento alimentar. Rev Nutr. 1999; 12(1):33-41.

43. Hansen BC. Symposium: Calorie restriction: effects on body composition, insulin signaling and aging. American Society for Nutritional Sciences; 2001. p.900S-02S.

44. Dietz WH. Does hunger cause obesity? Pediatrics. 1995; 95:766-67.

45. Townsend MS, Peerson J, Love B, Achterberg C, Murphy SP. Food insecurity is positively related to overweight in women. J Nutr. 2001; 131: 1738-45.

46. Olson CM. Nutrition and health outcomes associated with food insecurity and hunger. J Nutr. 1999; 129(2):521-24.

47. Cristofar S, Basiotis P. Dietary intakes and selected characteristics of women ages 19-50 years and their children ages 1-5 years by reported perception of food sufficiency. J Nutr Educ. 1992; 24(2):53-8.

48. Wilde PE, Ranny CK. The monthly food stamp cycle: shopping frequency and food intake decisions in an endogenous switching regression framework. Am J Agric Econ. 2000; 82(1):200-13.

49. Williams GC, Grow VM, Freedman ZR, Ryan RM, Deci EL. Motivational predictors of weight loss and weight-loss maintenance. J Pers Soc Psychol. 1996; 70(1):115-26.

Recebido para publicação em 25 de fevereiro de 2002 e aceito em 2 de julho de 2004. 